\title{
Smart IOT Based Healthcare Monitoring and Decision-Making System Using Augmented Data Recognition Algorithm
}

\author{
Annamalai.M" ${ }^{1}$, Dr. X. Mary Jesintha ${ }^{2}$, Dr.M.Nithya ${ }^{3}$ \\ ${ }^{1}$ Research Scholar, Department of Computer Science, Bharathiar University, Coimbatore. \\ ${ }^{2}$ Research Supervisor, Guest Lecturer, Department of Computer Science, Periyar University, Salem. \\ ${ }^{3}$ Professor, Department of Computer science and engineering, Vinayaka missions Kirupananda Variyaar Engineering \\ College, Salem,
}

Article History: Received: 10 January 2021; Revised: 12 February 2021; Accepted: 27 March 2021; Published online: 10 May 2021

\begin{abstract}
Many medical errors occur because people are in charge of patients or elderly medications by handling large amounts of medications every day. This work consists of designing and establishing a pillbox prototype intended to address this shortcoming in medical areas. It can be used separately from the medication itself and other advanced features provided with this device by the hospital or retirement home. This medication pack aims to take most medications or vitamin supplements or stimulants that deal with over-salting or over-the-counter patients. The proposed smart pillbox contains a program that enables medical caregivers or clients to determine the pill size and timing of pills and service routine each day. In this research work, the Augmented Data Recognition (ADR) algorithm is also used to monitor humans' health conditions. Initially, the UCI dataset is used for training and validation of the proposed ADR algorithm. The heart rate, blood pressure and temperature of the patient have carried during the testing phase via the Internet of Things (IoT) setup. The testing phase estimates any abnormalities in the health status based on the information obtained by the sensor collected by the population structure. Statistical analysis is based on data obtained from a cumulative cloud from IoT devices to estimate percentage accuracy.
\end{abstract}

Keywords: Medication pill, Augmented Data Recognition, Internet of Things, UCI dataset

\section{INTRODUCTION}

Many medical errors occur because individuals are in the care of the patient or the elderly are dealing in large quantities of pills every day. This work includes creating the pillbox model using IoT and a smart health monitoring plan, design, and ADR security algorithm [1], [2]. This work is intended to address this shortcoming in the medical field. It is also not intended to be used by many other advanced medical or retirement homes, with this device unable to sort by itself [3]. The proposed Smart Health Monitoring system is in patients taking medications or vitamin supplements or caregivers who are more salty or targeted patients [4].

In this way, medical caregivers or clients can set data for nine specific tablets. Remind clients or patients to take pills using pillbox sound and light [5] when no tablet time is specified. The tablets to be taken will be displayed through an android application arranged by the alert patient. On the other hand, a regular pillbox basket is required each day or dependably [6] with customers or security guards. This work introduces a new machine learning-based IoT system to overcome the conventional methods' drawbacks.

The proposed framework gives a stage for monitoring and regulating patients by the utilization sensor network. The design comprises both hardware and software programming sections. The hardware part incorporates a Heart rate sensor, Temperature sensor, Blood Pressure sensor and Raspberry Pi board. The processing stages are as per the following: medication pill remaining portion, an assortment of sensor esteems, storage of data in the cloud and the examination of the data stored in the cloud to check for anomalies in the health condition. Because of the anomalies, the pill and dosage esteems are refreshed.

\section{LITERATURE SURVEY}

Some important work in medical science has been done using IoT to monitor patient health. The work in this area is as follows.

Various strategies exist to invasively and noninvasively overview the heart rate and inward warmth level. Noninvasive procedures have demonstrated accurate and supportive outcomes for the customers [7], [8]. It is also said to work with health patients [9] to give extraordinary room conditions. A couple of estimates such as room humidity and the level of all gases such as $\mathrm{CO}$ and $\mathrm{CO} 2$ can be chosen ideas in-room. Toxic gases and certain humidity levels are particularly harmful to patients [10], [11], so it's necessary to monitor. The room humidity should be someplace in the scope of 30 and $65 \%$ for ideal help. A couple of assessments [12] are done particularly for a smart home, not for devoted healthcare.

Over the past decade, numerous IoT-based health care applications have been seeing significant advancements [13, 14]. Researchers have been chipping away at an IoT execution reference model. In [15], proposed a standard structure for improving IoT applications, similar to smart healthcare. According to this article, the design of an IoT application is guided by three primary concerns of view: cloud-focused IoT, network-focused IoT and data-focused IoT. These three perspectives affected distinctive IoT applications to be executed.

In [16], an IoT-focused investigation framework was utilized to demonstrate a client electronic gadget. The system settles with the customer if their health is above or below the reach of the standard. Accumulated data went to the cloud using an application and was sent off by the Field Programmable Gate Array (FPGA). Regular data were resolved and arranged to use FPGA; Furthermore, the active polynomial conditions were set on the 
patient's wearable IoT device [17]. The strategy laid in the fuzzy classifier improvement construed the over-thetop condition of contaminations with high precision [18]. The FPGA execution of the fuzzy classifier gave low execution time stood out from K-Nearest Neighbor (KNN), Support Vector Machine (SVM), decision tree, just as Naive Bayes [19-21]

\section{PROPOSED ADR BASED HEALTH MONITORING AND DECISION-MAKING SYSTEM}

The proposed system includes an installed health monitoring device and an automated decision-making system to improve medication and decision-making. Both tools can communicate with each other by installing a device-verified handshake protocol personal area network. They are designed for medical updating, delivering an interface and details and physical signal recording settings to access. Doctors can access the interface through a web interface to change the system's touch screen and drug sharing settings. Transferring records from the monitoring device to the system. Then, the allocation system's distribution encrypts the data using the control algorithm, namely, Augmented Data Recognize.

The cloud framework is intended for patient security and assurance. Hence, the transferred data is stored in the patient file and moved aside from government issues that can't be accessed. Two analytical methods are presented to help individual or short-term and long-term results to categorize data on analytical hygiene compiled for all individuals in the cloud. Records are integrated with the electronic health record to manage the patient's history. Provides an interface for further analysis of the proposed system reports and decision-making. Figure 1 summarizes the architecture presented by ADR based on Smart Health Monitoring and Pill Remedies with decision-making support.

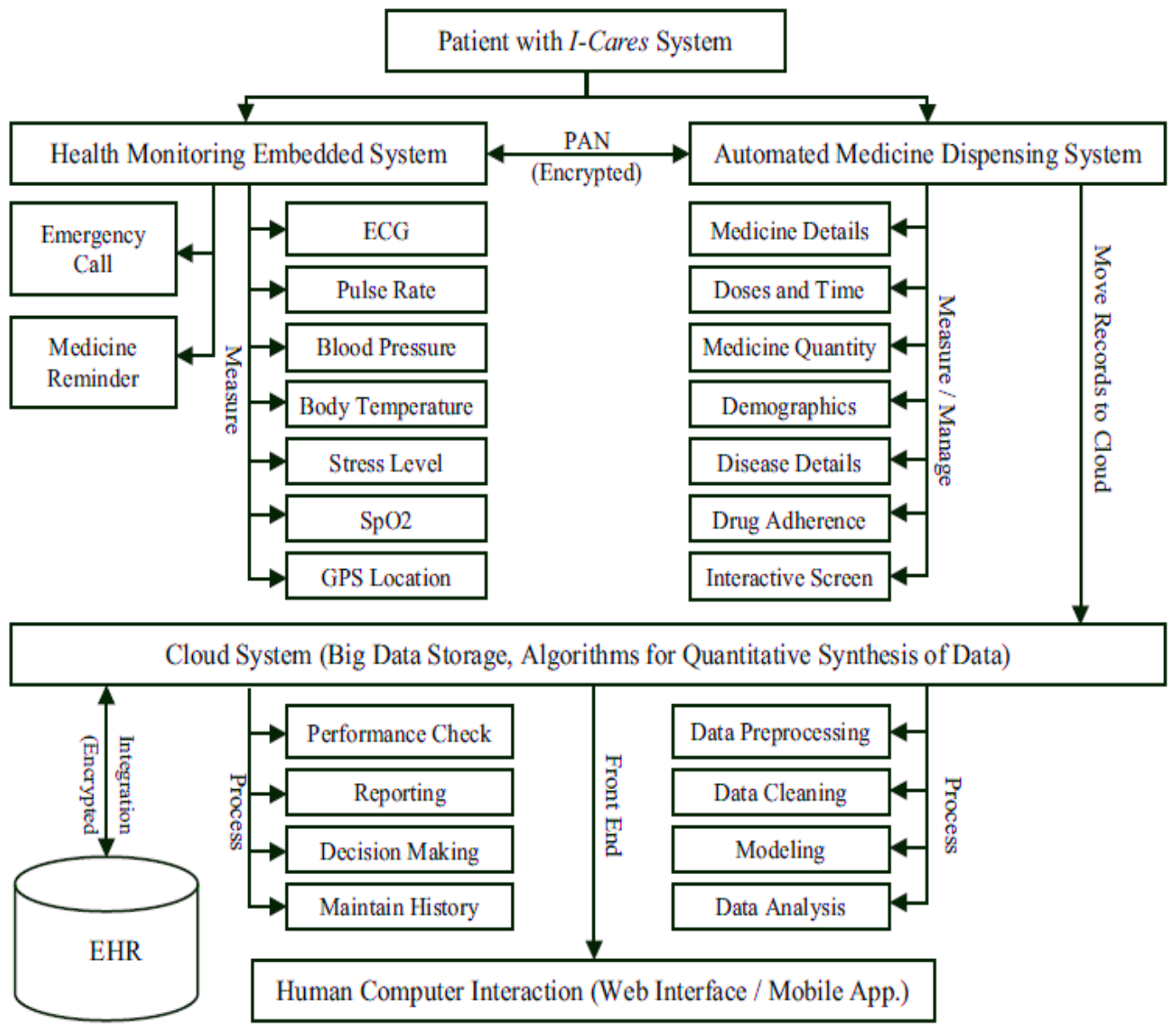

Figure 1 Architecture of ADR-based smart healthcare monitoring and pill remainder.

\subsection{ADR based Healthcare Monitoring System}

The proposed ADR health monitoring framework including sensors that can measure heart rate, blood pressure, internal heat level, anxiety, perspiring, and peripheral capillary oxygen saturation (SpO2). Nonetheless, this framework can be increased. A controller (program log) permits patients to add new sensors and sensor settings, understanding frequency, threshold level, etc., after login. This plug-and-play system makes it easy to configure the patient. The monitoring system, which sends these measurements to the Smart Medical Distributor Cloud, sends scalable. Remind him/her to schedule a medical dose for her by contacting the patient alarm monitoring system intelligent medical communication system. 

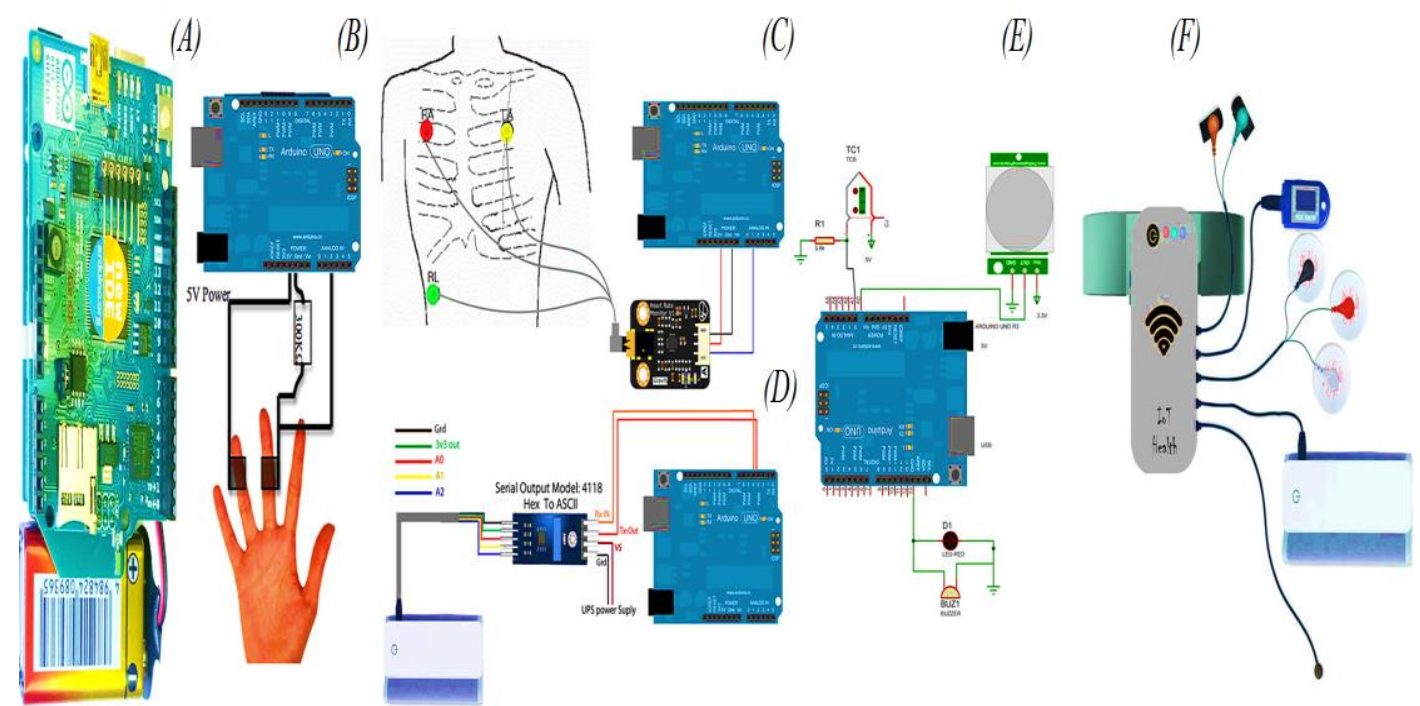

Figure 2. Prototype design of Proposed System

Figure 2 shows the proposed convenient health checking framework and an illustration of sensor associations with an Arduino microcontroller. The small health monitor was provided with basic selection screens sensors and an Arduino chip (to pick up emergency personnel and send to the patient's location in case of emergency).

The monitoring system is equipped to send readings to the pharmacist, send readings to the Wi-Fi cloud and emergency reports. The reading program shows the total reading range for the individual sensors at the maximum level. More or less, reading the line shows examples. In which an emergency flag has flown, the variance for readings is further indicated. Based on this purpose, Arduino can determine whether an emergency event is active or whether medical personnel needs immediate attention.

\subsection{ADR based Medicine Pill Reminder System}

The proposed ADR system includes a smart medicine reminder and delivery system. This system makes smart decisions within clinical prospect parameters and allows emergency priorities to lower priorities in out-oftable medicine detail. Figure 3 shows the model and model interior parts that may have been used separately. The proposed Smart Medicine Bill Reminder and Health Monitoring system will be integrated through the Personal Area Network. The proposed system includes an interactive LCD screen to configure monitoring device (frequency-determining measurements and other features), pharmaceutical system and communication number sensors with Smart Medical Distributor to give users detailed feedback on drug side effects.

(A)

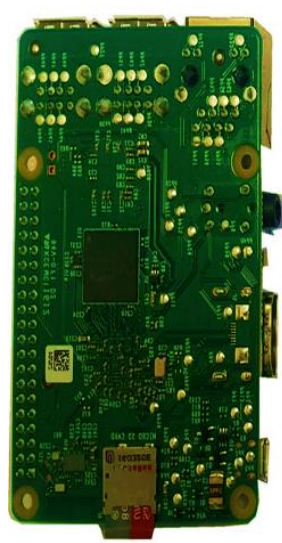

(B)

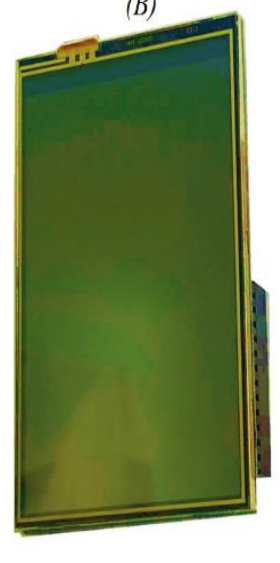

(C)
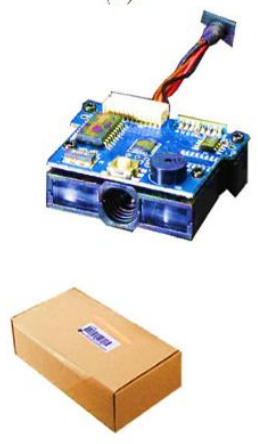

(D)

(E)

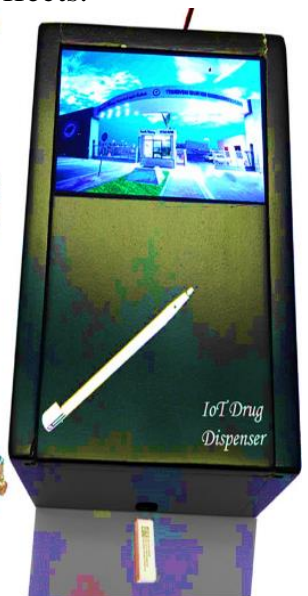

Figure 3 Smart Medicine reminder and dispensing System

However, this LCD screen also has an intelligent visual interface that gets the specialist's dosage recurrence. A Raspberry Pi 3 is utilized as a regulator in this framework. It is empowered for associating a Wi-Fi health screen and a cloud worker. Giving automated medication is smart since it speaks with both the patient and the specialist. Healthcare providers can exchange pharmaceutical and delivery frequency information remotely. The device physically ensures that the patient can be contacted as he/she receives the required reminders and dosage.

\subsection{Analysis of Cloud Storage}

Cloud systems are the best solution for a large number of applications located in different geographical locations. Measurements are sent to the cloud by both ADR monitoring, smart medicine pill reminder and delivery system. Long-term clinical assessment is a significant part of the proposed framework. It very well may be planned by tolerant requirements, e.g., week by week, month to month, quarterly or yearly, to anticipate any disease in the 
future. The long-term diagnostic algorithm is designed to predict diseases that may occur in the future based on the system's features using a health test as ADR power. In a classification problem, the specific possibilities to measure are based on different features

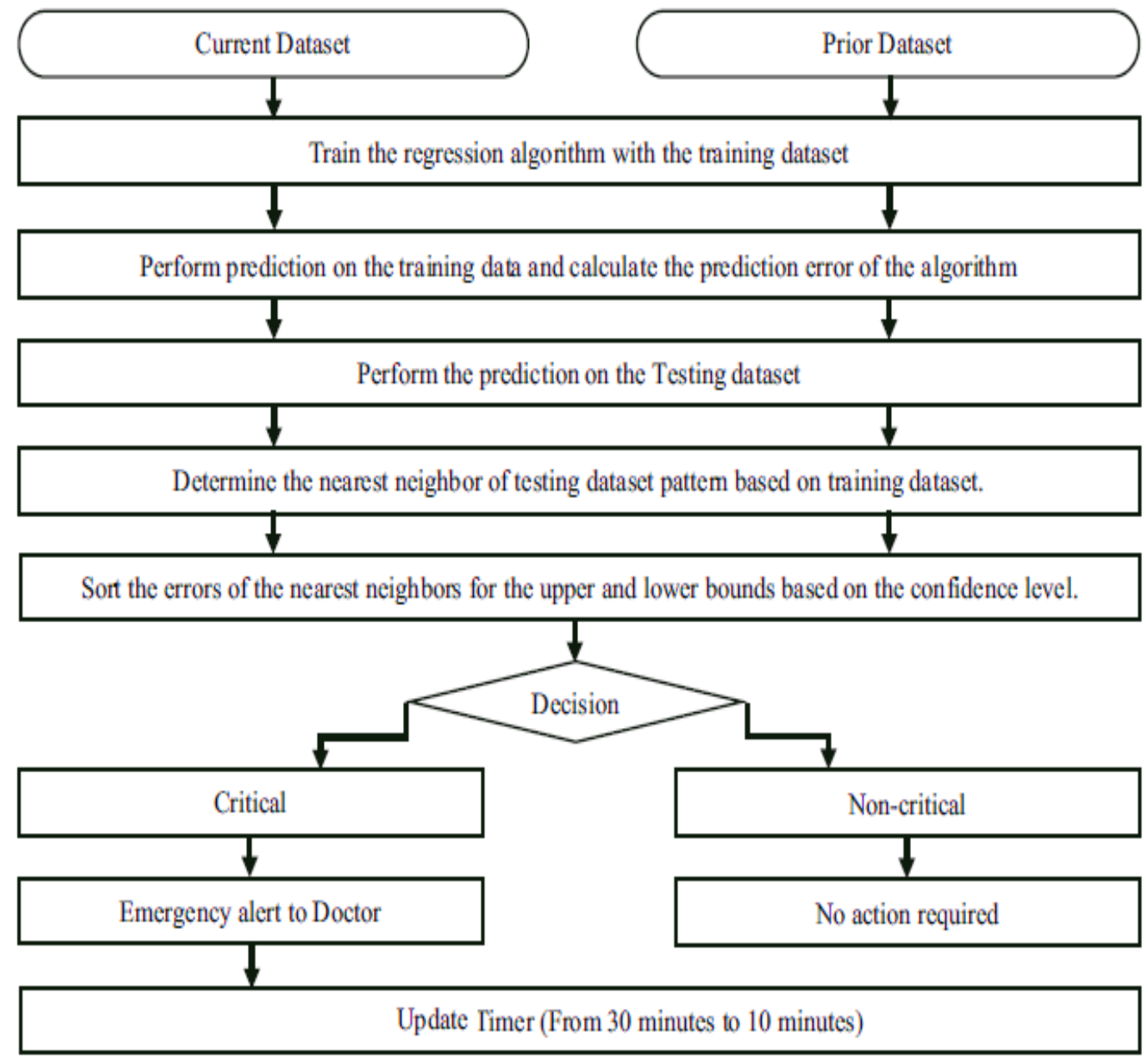

Figure 4 short-term analysis and emergency alert system

Measuring the signal of the human body is an important and arduous task, especially for short-term important health predictions. Forward-looking trust requires an emergency call based on the doctor or nursing staff's lower limit and upper limit. Figure 4 shows the proposed methodology for short-term health prognosis. The regression algorithm proposes systematic training to popularize various health-related aspects of training data in the first step. After the training is over, the classification algorithm is calculated based on the predicted value applied to the prediction error based on the information-based training prediction. In the next step, the model is classified and defined as the upper and lower limits of comparison based on the ADR algorithm.

\subsection{Augmented Data Recognize for IoT Security Algorithm}

Medical management is an organization that can be used to monitor the tools of today. ADR Machine learning and significant data detection sensors are sensitive to loading information. This innovation helps to control business gadgets

i. For the first time, users must log in to enter the system.

ii. The essential elements after verifying the user's credentials.

iii. Here it is stored as a cloud server for all user information.

Cloud server consists of the following:

i. Admin (Alert): Control through the system and send a cloud server message.

ii. Technician: The server can solve it when the cloud problem occurs.

iii. Remote Monitoring: Works automatically using sensors.

iv. Decision Maker: It will decide to send a warning via SMS / E-mail.

ADR Control Procedure for Case: (Medical Working Environment)

Step 1: Booting the KERNEL

Step 2: Connecting the sensors

Step 3: Reading the Sensor values from the Terminal

Step 4: Reading the sensors from the program

Program Algorithm:

Step 1: Install one pilot wire channels and interact with the sensor

Step 2: Set the time interval 
Step 3: Set the drivers

Step 4: Create the output file to record the temperature

Step 5: Create a variable for sensors

Step 6: Sensor Video on Cloud Sensor Show -Display

Different sensors are used to read the quality parameters and send the cloud-enabled system using the Raspberry Pi data controller. Programs using internet programming language are based on proposed wiring and IoT growth based on proposed controller processing. The error rate is derived from the flow rate difference between the different parameters.

\section{RESULT AND DISCUSSION OF ADR BASED HEALTHCARE MONITORING SYSTEM}

This section presents the result and discussion of the proposed ADR-based smart healthcare management system and medicine pill reminder using IoT. The following figure 5 shows the hardware prototype model of the proposed system.

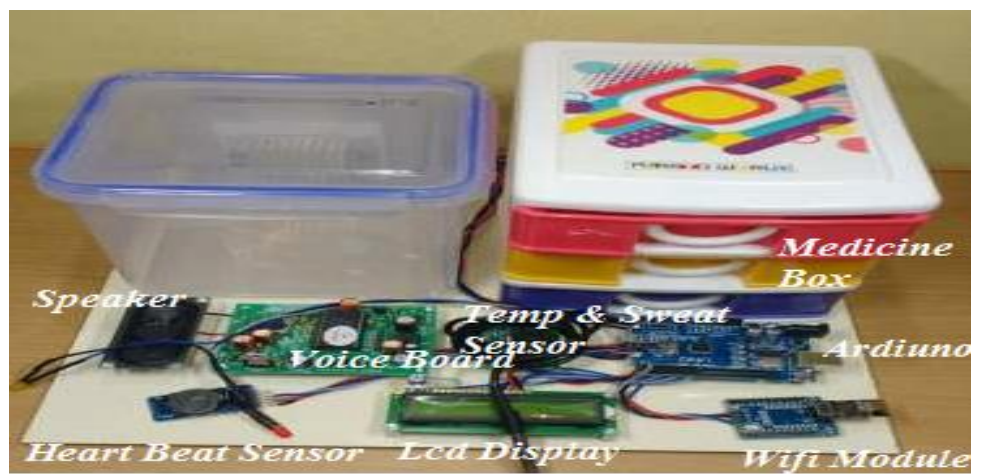

Figure 5 Prototype Model of Proposed System

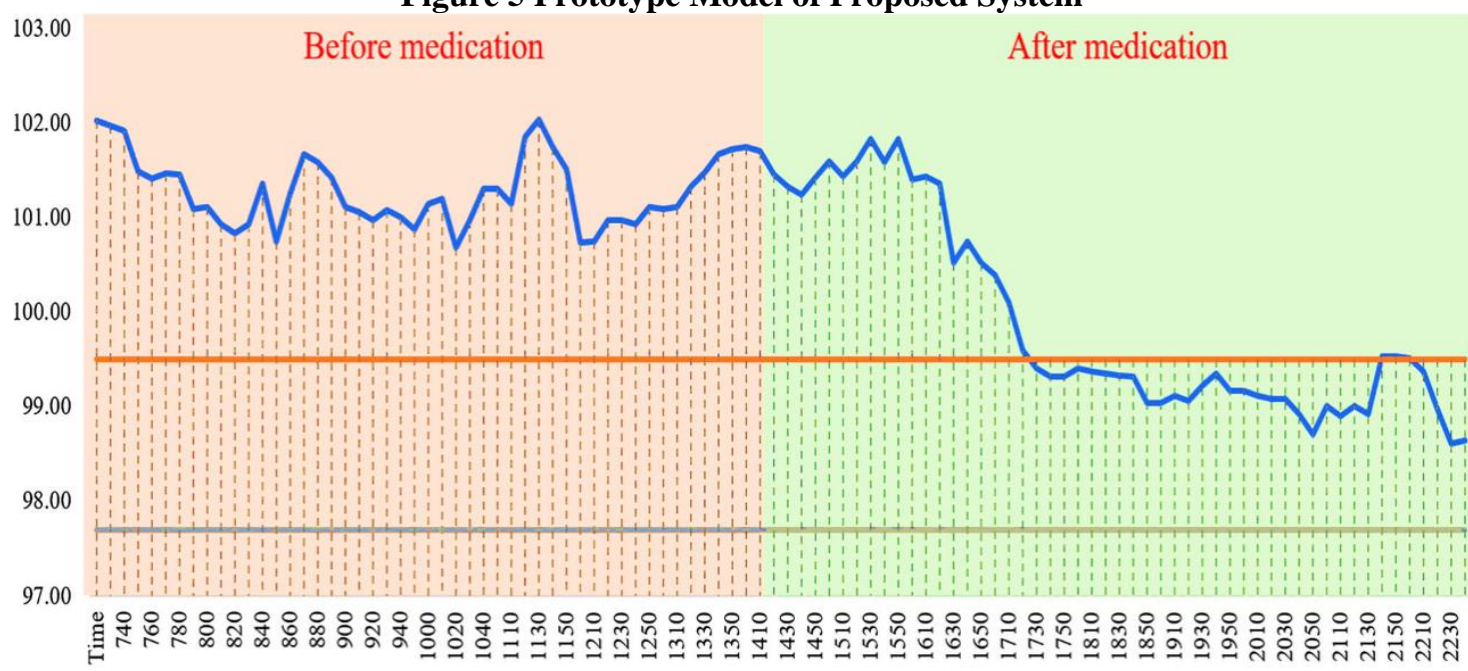

- Calculated Body Temperature (F) $\quad$ Normal Temperature (Max) $\quad$ Normal Temperature (Min)

Figure 6 measurement of Body temperature

Figure 6 shows the body temperature pattern captured by the proposed System for medication taken at 12:10, and it shows body temperature before and after taking the drug. As shown in these results, real-time body temperature is measured by the system's sensor. A clever set of rules based on the recommended amount of flu medication and a specific time setting. Even after taking the drug, the sensor takes measures and maintenance to ensure that the temperature drops to an acceptable leve1 temperature.

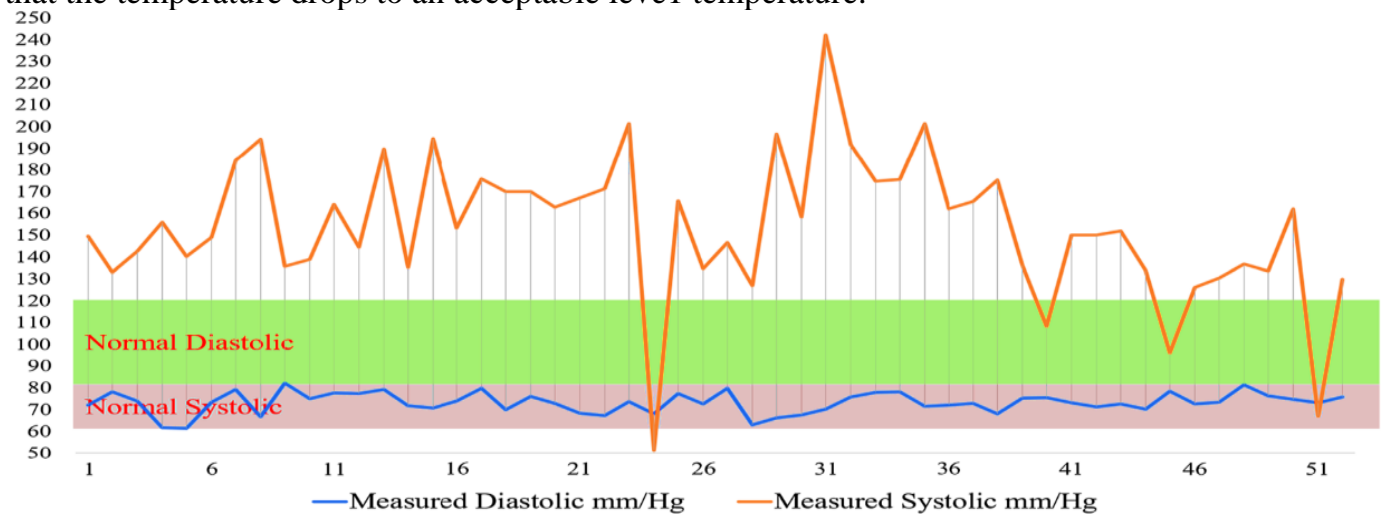

Figure 7 Measurement of Blood Pressure Measurement 
Figure 7 shows the real-time measurement model, along with warnings in value in the case of blood pressure (contraction and dilation) exceeding a normal threshold. These warnings are further categorized as warning patients to take medication or alert/call for medical assistance in serious cases.

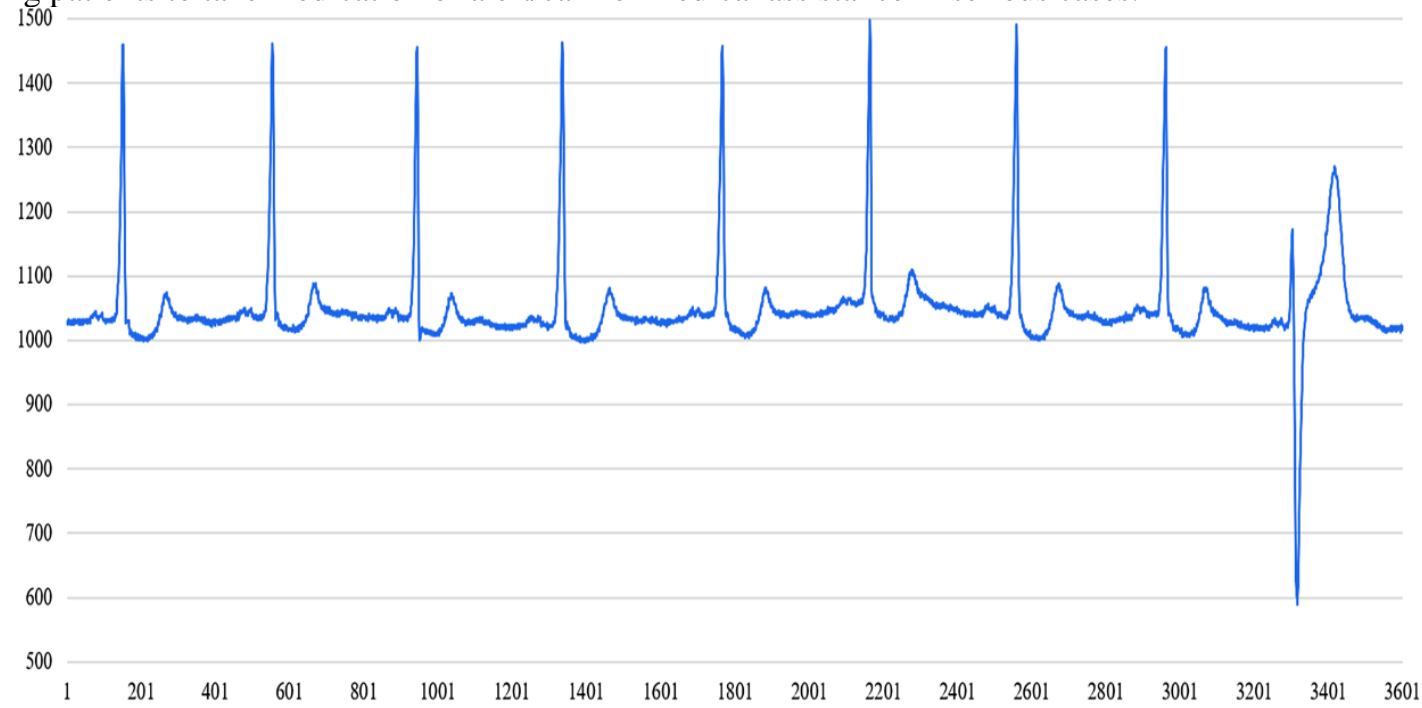

Figure 8 Measurement of ECG

Figure 8 shows the ECG model measurement. The system can measure ECG well, and take necessary urgent measures, and provide a web interface for patients and medical staff.

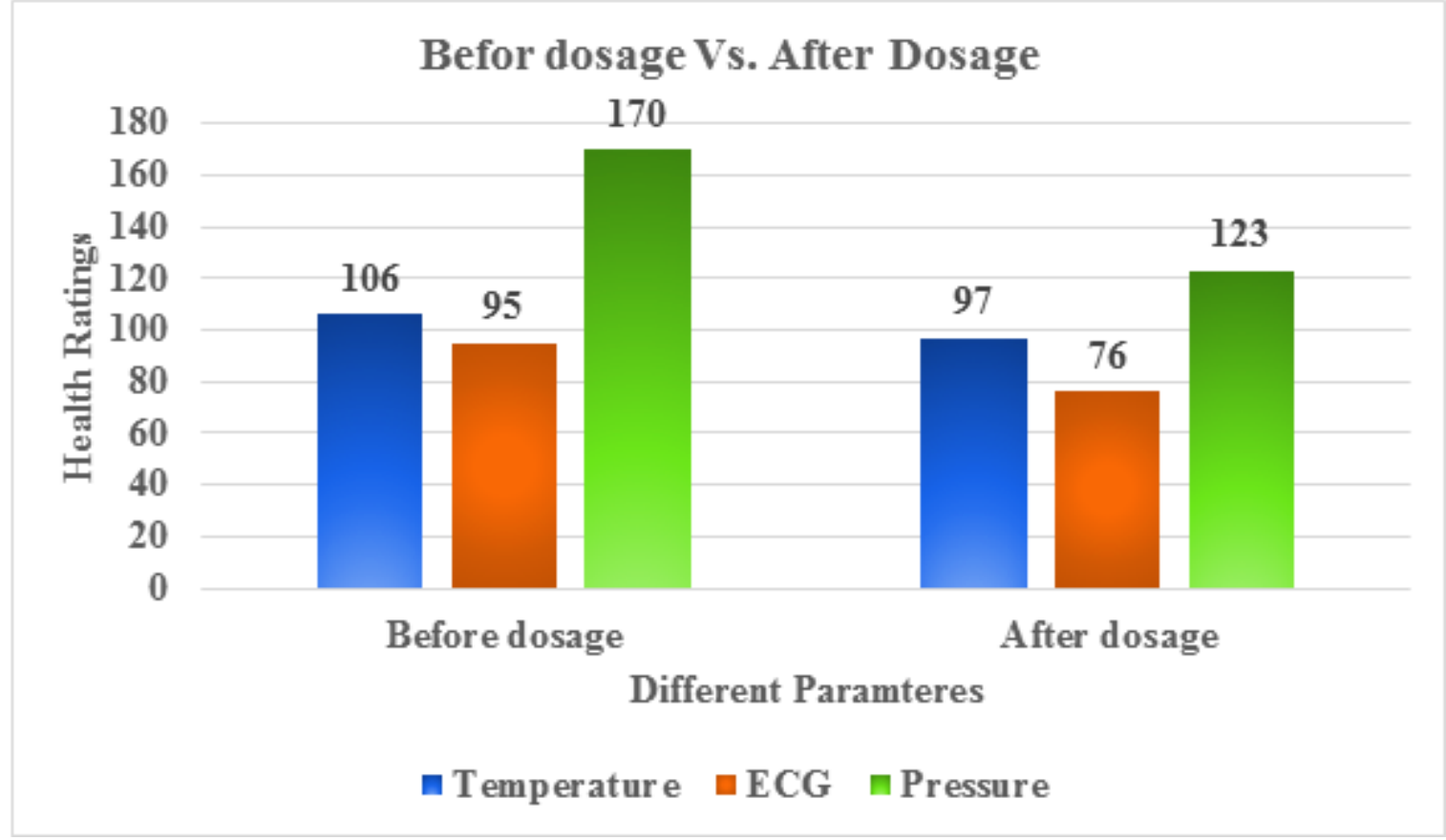

Figure 10. Performance analysis after dosage is given.

Above, figure 10 shows the healthcare results of before dosage and after dosage given. This analysis clearly shows that the health is controlled and maintained at a normal stage after giving the dosage.

\subsection{Performance analysis of ADR}

This section discusses the results and performance analysis of the proposed system. The Augmented Data Recognize security algorithm-based IoT system and programming for medical evaluation structure have been analyzed. The performance of the proposed system is compared with the following existing methods.

a) Flexible Route Based Congestion Avoidance (FRBCA)

b) Distributed Route-Aggregation (DRA)

c) Registry Service Selection (RSS)

Table 1 Comparison of security performance-ADR

\begin{tabular}{cccc}
\hline Algorithms & $\begin{array}{c}\text { Brute Force } \\
\text { Attack }(\%)\end{array}$ & $\begin{array}{c}\text { Web-based } \\
\text { Attack }(\%)\end{array}$ & $\begin{array}{c}\text { Unknown Attack } \\
(\%)\end{array}$ \\
\hline FRBCA & 074 & 072 & 071 \\
\hline DRA & 081 & 079 & 076 \\
\hline RSS & 085.5 & 080.3 & 082.2 \\
\hline
\end{tabular}




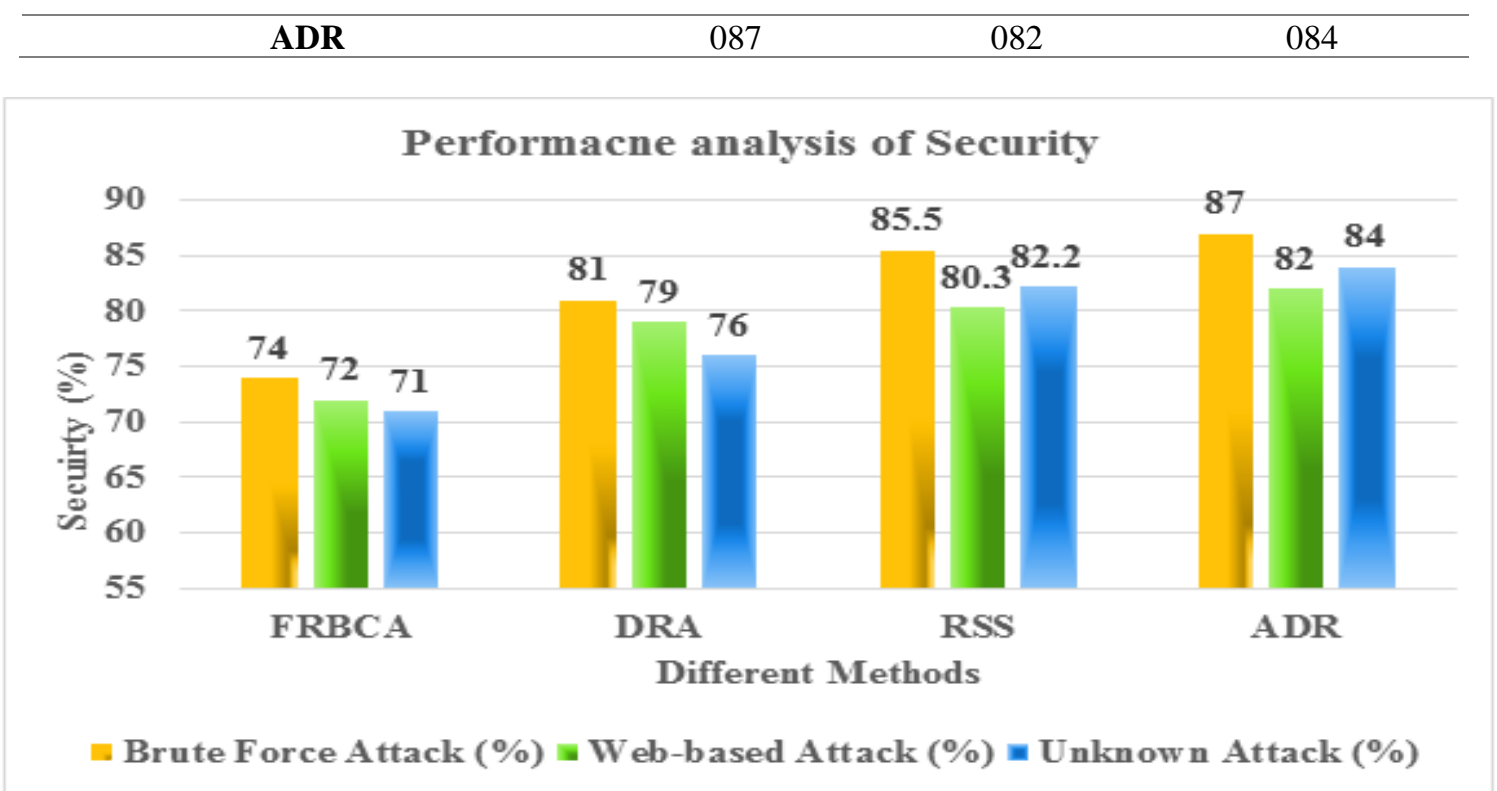

Figure 11 Comparison of security level Using ADR

Figure 11 and Table 1 show the level of safety to the comparative result, and it should be noted that the proposed method produced a higher level of security than others. As compared with existing methods, the proposed ADR gives the best result against brute force attack (87\%), web-based Attack (82\%) and unknown Attack $(84 \%)$.

Table 2 Performance Analysis of Service utilization

$\begin{array}{llllll}\text { Algorithms } & 1 \text { million } & 2 \text { million } & 3 \text { million } & 4 \text { million } & 5 \text { million }\end{array}$

\begin{tabular}{cccccc}
\hline FRBCA & 85 & 80.5 & 78.05 & 77.12 & 74.26 \\
\hline DRA & 87.96 & 85.14 & 81.01 & 76.56 & 75.89 \\
RSS & 93.12 & 91.02 & 89.56 & 87.24 & 85.10 \\
\hline ADR & 95.23 & 94.36 & 94.23 & 90.12 & 91.23 \\
\hline
\end{tabular}

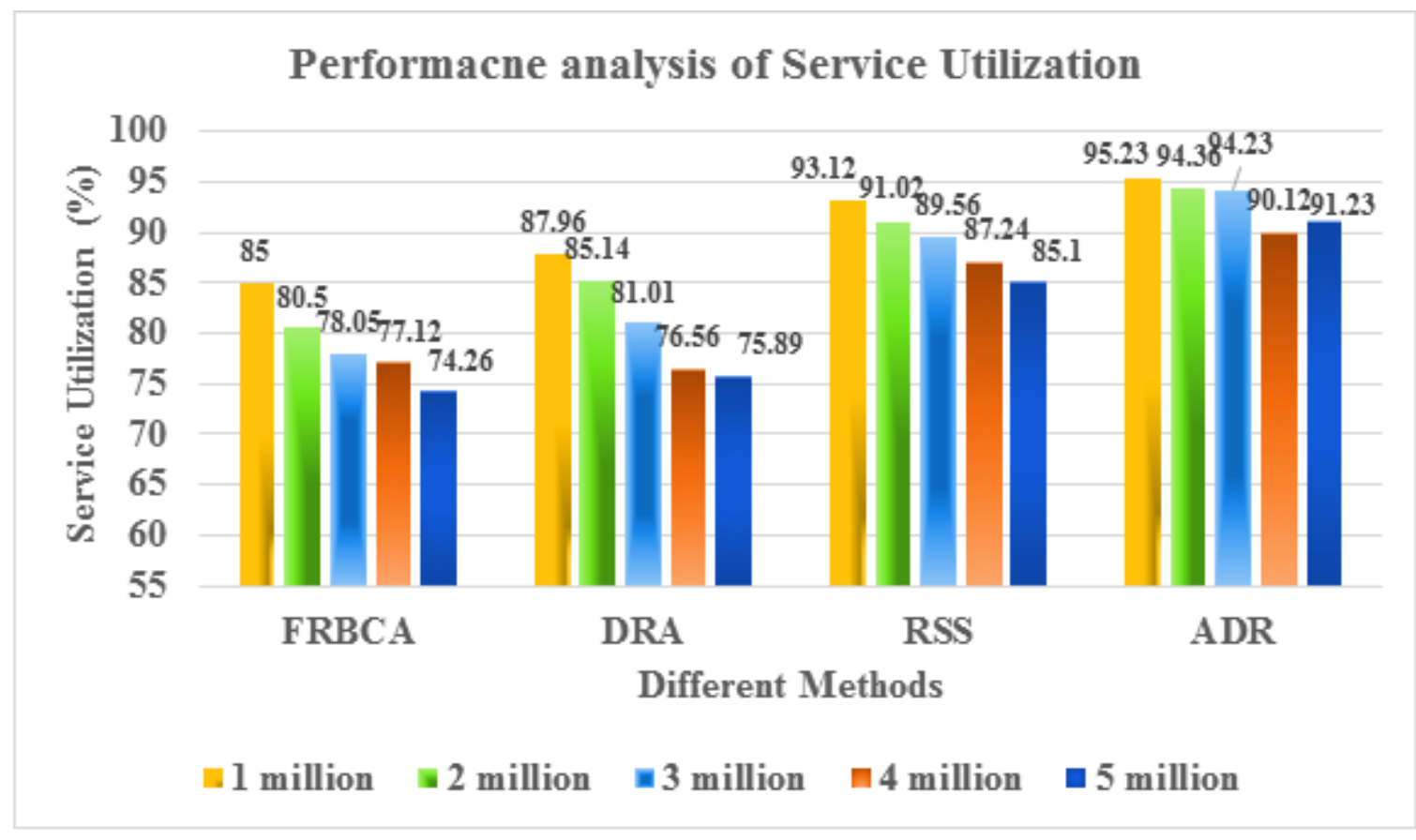

Figure 12 Performance analysis - service availability routine using RSS and ADR 
Figure 12 and Table 2 show the availability based on the service in the Cloud area. Compared with existing methods, the proposed ADR method obtains perfect results in every operating state.

Table 3 Time Complexity Analysis - ADR

\begin{tabular}{cccc}
\hline Algorithms & $\mathbf{1 0}$ Locations & $\mathbf{5 0}$ Locations & 100 Locations \\
\hline FRBCA & 3.2 & 2.9 & 2.5 \\
\hline DRA & 2.5 & 2.1 & 1.75 \\
\hline RSS & 2.1 & 1.95 & 1.4 \\
\hline ADR & 2.01 & 1.85 & 1.3 \\
\hline
\end{tabular}

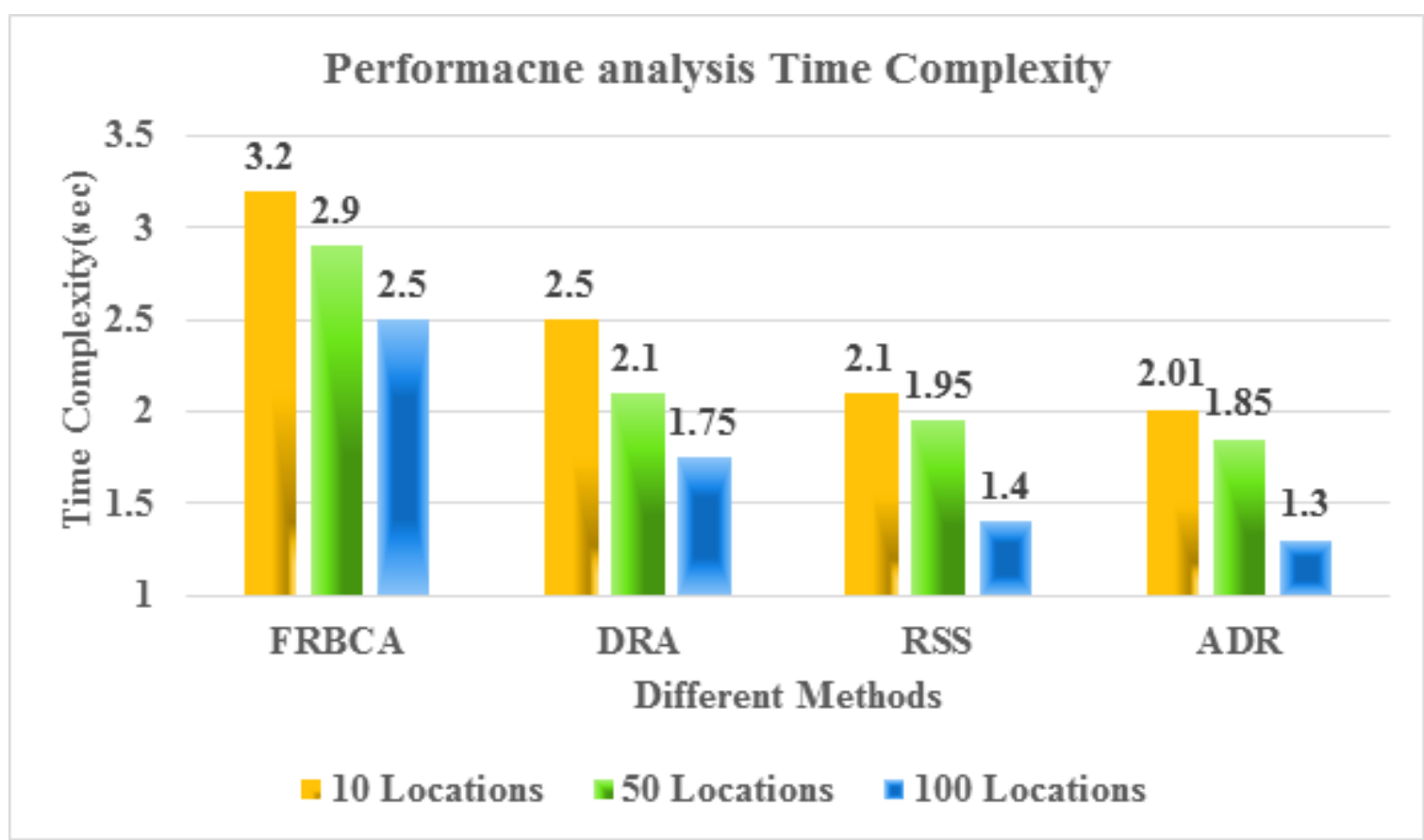

Figure 13 Time complexity of proposed and existing approaches

Figure 13 and Table3 show the performance analysis of time complexity using different methods. This comparison clearly says that as compared with existing methods, the proposed ADR gives a low time complexity value against conventional methods. As compared with existing methods, the proposed ADR method takes minimum time complexity. The time complexity of ADR is $2.01 \mathrm{sec}$ from 10 locations, the time complexity of ADR is $1.85 \mathrm{sec}$ from 50 locations, and the time complexity of ADR is $1.3 \mathrm{sec}$ from 100 locations.

\section{CONCLUSION}

A novel approach was introduced, and IoT-based smart health monitoring and decision-making systems are evolving in this work. This comprehensive theoretical framework is then the embodiment of an innovative prototype that demonstrates the practical functioning of the proposed smart healthcare system. The system provides ongoing analysis and monitoring of a patient's health status, side effects of medications, side effects, and real-time automated emergency intervention that can ultimately save lives. The system is equipped with a small wearable design sensor to measure patients 'vitality. The proposed system is connected through a private area network to smart drug providers. Both patient measurements, dose and frequency, are sent to a cloud-based health information system. The system can provide reports and dates, including results, recommended by physicians to determine drug dosages.

\section{REFERENCES}

1. Islam, M.M., Rahaman, A. \& Islam, M.R. Development of Smart Healthcare Monitoring System in IoT Environment. SN COMPUT. SCI. 1, 185 (2020). https://doi.org/10.1007/s42979-020-00195-y

2. Prajoona Valsalan, Tariq Ahmed Barham Baomar, Ali Hussain Omar Baabood. IOT BASED HEALTH MONITORING SYSTEM. http://www.jcreview.com/?mno=96095 [Access: April 10, 2021]. doi:10.31838/jcr.07.04.137

3. Taiwo O, Ezugwu AE. Smart healthcare support for remote patient monitoring during covid-19 quarantine. Inform Med Unlocked. 2020;20:100428. doi:10.1016/j.imu.2020.100428

4. Taştan, Mehmet. (2018). IoT Based Wearable Smart Health Monitoring System. Celal Bayar Üniversitesi Fen Bilimleri Dergisi. 343-350. 10.18466/cbayarfbe.451076.

5. R. Alekya, Neelima Devi Boddeti, K. Salomi Monica, Dr.R. Prabha, Dr.V. Venkatesh. "IoT Based Smart Healthcare Monitoring Systems: A Literature Review". European Journal of Molecular \& Clinical Medicine, 7, 11, 2021, 2761-2769. 
6. Rahaman, A., Islam, M.M., Islam, M.R., Sadi, M.S., Nooruddin, S. (2019). Developing IoT-based smart health monitoring systems: A review. Revue d'Intelligence Artificielle, Vol. 33, No. 6, pp. 435-440. https://doi.org/10.18280/ria.330605

7. Sunny, Saina \& Kumar, S.. (2017). IoT Based Non-Invasive Glucometer Using Optical Method. International Journal of Advanced Research in Computer Science and Software Engineering. 7. 42. 10.23956/ijarcsse.v7i9.409.

8. Ray, Partha \& Thapa, Nishant \& Dash, Dinesh \& De, Debashis. (2019). A novel implementation of IoTbased non-invasive sensor system for real-time monitoring of intravenous fluid level for assistive ehealthcare. Circuit World. 45. 109-123. 10.1108/CW-01-2019-0008.

9. Varghese 1, G.Swathi2, Non-Invasive Haemoglobin Measurement System Using IoT And Mobile Devices, International Journal of Innovative Research in Science, Engineering and Technology (IJIRSET), Volume 9, Issue 4, pp. 1306-1309, April 2020

10. Yang, CT., Chen, ST., Chang, CH. et al. Implementation of an Environmental Quality and Harmful Gases Monitoring System in Cloud. J. Med. Biol. Eng. 39, 456-469 (2019). https://doi.org/10.1007/s40846018-0383-0

11. Afsar, M.F., Rafiq, M.A., Tok, A.I.Y. et al. Humidity and selective oxygen detection by $\operatorname{Ag}_{2} \mathrm{~S}$ nanoparticles gas sensor. J Mater Sci: Mater Electron 30, 10117-10127 (2019). https://doi.org/10.1007/s10854-019-01347-4

12. Islam, Md \& Rahaman, Ashikur \& Islam, Rashedul. (2020). Development of Smart Healthcare Monitoring System in IoT Environment. SN Computer Science. 1. 185. 10.1007/s42979-020-00195-y.

13. Jaiswal K., Anand V. (2021) A Survey on IoT-Based Healthcare System: Potential Applications, Issues, and Challenges. In: Rizvanov A.A., Singh B.K., Ganasala P. (eds) Advances in Biomedical Engineering and Technology. Lecture Notes in Bioengineering. Springer, Singapore. https://doi.org/10.1007/978-981-15-6329-4_38

14. Baker, Stephanie \& Xiang, Wei \& Atkinson, Ian. (2017). Internet of Things for Smart Healthcare: Technologies, Challenges, and Opportunities. IEEE Access. PP. 1-1. 10.1109/ACCESS.2017.2775180.

15. Islam, S. M. Riazul \& Kwak, Daehan \& Kabir, Md. Humaun \& Hossain, Mahmud \& Kwak, Kyung. (2015). The Internet of Things for Health Care: A Comprehensive Survey. IEEE Access. 3. 678-708. 10.1109/ACCESS.2015.2437951.

16. Rahaman, Ashikur \& Islam, Md \& Islam, Rashedul \& Sadi, Muhammad \& Nooruddin, Sheikh. (2019). Developing IoT Based Smart Health Monitoring Systems: A Review. Revue d intelligence artificielle. 33. 435-440. 10.18280/ria.330605.

17. Rao, Muzaffar \& Newe, Thomas \& Grout, I.A. \& Lewis, Elfed \& Mathur, Avijit. (2015). FPGA-based Real time secure body temperature monitoring suitable for WBSN. 10.1109/CIT/IUCC/DASC/PICOM.2015.22.

18. Mahendran, Rakesh \& v, Parthasarathy. (2020). A secure fuzzy extractor-based biometric key authentication scheme for a body sensor network on the Internet of Medical Things. Computer Communications. 153. 10.1016/j.comcom.2020.01.077.

19. Oyaga, Jaime \& Pozo, Francesc \& Tibaduiza Burgos, Diego \& Anaya, Maribel. (2017). A Sensor Data Fusion System Based on k-Nearest Neighbor Pattern Classification for Structural Health Monitoring Applications. Sensors. 17. 1-26. 10.3390/s17020417.

20. Ben Rejab, Fahmi \& Nouira, Kaouther \& Trabelsi, Abdelwahed. (2014). Real Time SVM for Health Monitoring System. 10.1007/978-3-319-09891-3_28.

21. Costa K, Ribeiro P, Carmargo A, Rossi V, Martins H, Neves M, Fabris R, Imaisumi R, Papa JP (2013) Comparison of the techniques decision tee and MLP for data mining in SPAMs detection in computer networks. Proceedings of the 3rd international conference on innovative computing technology, pp 344$--348$ 